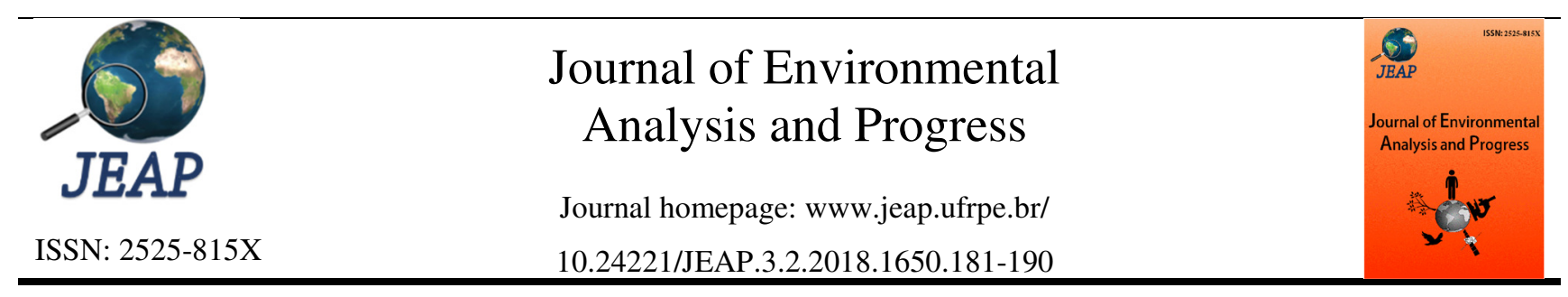

\title{
A Floresta Nacional (FLONA) da Restinga de Cabedelo e a sua influência no clima urbano da cidade de Cabedelo-PB
}

\section{The National Forest (FLONA) Restinga of Cabedelo and its influence on the urban climate of the city Cabedelo-PB}

\author{
Anne Falcão de Freitas ${ }^{\mathrm{a}}$, Joel Silva dos Santos ${ }^{\mathrm{a}}$, Bartolomeu Israel de Souza ${ }^{\mathrm{a}}$, Ingrid Almeida ${ }^{\mathrm{a}}$, \\ Nayara Silva Lins de Albuquerque ${ }^{b}$ \\ a Universidade Federal da Paraíba-UFPB. Programa de Pós-Graduação em Desenvolvimento e Meio Ambiente- \\ PRODEMA, Cidade Universitária, s/n, Castelo Branco, João Pessoa-PB. E-mail: annefalcao@ gmail.com. \\ ${ }^{\text {b }}$ Universidade Federal de Pernambuco-UFPE. Programa de Pós-Graduação em Biologia Vegetal-PPGBV, Av. Prof. \\ Moraes Rego, n. 1235, Cidade Universitária, Recife-PE.
}

\begin{tabular}{l}
\hline A R T I C L E I N F O \\
\hline Recebido 22 Set 2017 \\
Aceito 31 Jan 2018 \\
Publicado 02 Abr 2018
\end{tabular}

\begin{abstract}
A B S T R A C T
Considering the disorderly growth of the urban network in the metropolitan area of the city of Cabedelo-PB, the Atlantic Forest biome has been intensely fragmented, and alterations in the local microclimate are perceptible by the increase in temperatures. However, studies aiming to understand the role of this forest reserve in the climatic mitigation of the city are still non-existent. This study aimed to verify the relationship between FLONA and the microclimate of an urbanized area in the city of Cabedelo-PB. The research starts from the hypothesis of a thermal gradient between FLONA and the urbanized area. Initially, a bibliographic survey was carried out regarding the subject matter, followed by the definition of the experimental points located within the FLONA and in the urban network of the city of Cabedelo-PB. Considering analyzing the existence of a thermal gradient, air temperature, and relative humidity, it was performed measurements using thermohygrometers during the dry season (December 2016 to January 2017). Three collection points were allocated within the FLONA and a collection point in the city's urban network. The results reveal the existence of a thermal gradient that indicates a gradual attenuation of the temperature and increase of the humidity as one advance to the interior of the FLONA, highlights the role of vegetation cover in the urban climate mitigation of the city of Cabedelo-PB. It reinforces the importance of the ecosystem service provided by the Atlantic Forest fragments and its necessary conservation.
\end{abstract}

Keywords: Microclimate, thermal gradient, Atlantic forest.

\section{R E S U M O}

Considerando o crescimento desordenado da malha urbana na região metropolitana da cidade de Cabedelo/PB, o bioma de Mata Atlântica tem sido fragmentado intensamente, e alterações no microclima local, são perceptíveis pelo aumento das temperaturas. No entanto, estudos visando compreender o papel desta reserva florestal na amenização climática da cidade ainda são inexistentes. Este estudo objetivou verificar a relação entre a FLONA e o microclima de uma área urbanizada na cidade de Cabedelo/PB. A pesquisa parte da hipótese da existência gradiente térmico entre a FLONA e a área urbanizada. Inicialmente foi realizado um levantamento bibliográfico a respeito da temática em questão, seguido da definição dos pontos experimentais localizados dentro da FLONA e na malha urbana da cidade de Cabedelo/PB. Para analisar a existência de um gradiente térmico, foram realizadas coletas de temperatura e umidade relativa do ar, utilizando termohigrômetros durante o período de estiagem da região (dezembro 2016 a janeiro 2017). Três pontos de coletas ficaram alocados dentro da FLONA e um ponto de coleta na malha urbana da cidade. Os resultados revelam a existência 
de um gradiente térmico que indica uma atenuação gradual da temperatura e aumento da umidade à medida que se avança para o interior da FLONA, destacando também o papel da cobertura vegetal na amenização do clima urbano da cidade de Cabedelo-PB. Isto reforça a importância dos serviços ecossistêmicos prestados pelos fragmentos de Mata Atlântica e a sua necessária conservação.

Palavras-Chave: Microclima, gradiente térmico, Mata Atlântica.

\section{Introdução}

A população urbana mundial aumenta a cada ano e, por meio da sua expansão, desestabiliza os sistemas ambientais, causando diversos problemas, como poluição, fragmentação dos biomas e perda de biodiversidade. Sendo assim, uma das principais consequências é a diminuição dos serviços ecossistêmicos (Zhou et al., 2014; Petrisor et al., 2016; Yi et al., 2016).

Um dos biomas que mais sofre com essa expansão humana é a Mata Atlântica, a qual passou por um processo de fragmentação e perda de mais de $93 \%$ da área original, sendo, portanto, incluída nos hotspots mundiais, por apresentar uma grande diversidade biológica, elevado grau de endemismo e estar ameaçada (Myres et al., 2000).

Conforme esse ecossistema é fragmentado, ocorre perda de habitat e criação de "ilhas" com diferentes tamanhos (Saunders et al., 1991), os quais sofrem alterações bióticas e abióticas à medida que se aproxima da margem da mata, sendo denominado de efeito de borda (Ranta et al., 1998; Fahring, 2003; McDonald et al., 2013).

No que diz respeito ao efeito de borda, os fatores abióticos temperatura e umidade relativa do ar são as variáveis microclimáticas mais sensíveis de mudanças. Tais variáveis podem sofrer alterações (Chen et al., 1999; Amorim, 2011; Sampaio, 2011).

Ewers \& Bank-Leite (2013), em estudo sobre fragmento de Mata Atlântica, constataram que a cada aumento de $1{ }^{\circ} \mathrm{C}$ na temperatura na região urbanizada, na floresta aumenta apenas $0,38^{\circ} \mathrm{C}$, mostrando que áreas arborizadas atuam na amenização climática, e que é de suma importância a manutenção desse ecossistema para contribuir com a diminuição do calor gerado pelas áreas urbanas.

$\mathrm{Na}$ cidade de Cabedelo, localizada na Paraíba, o processo de expansão urbana se deu ao longo da rodovia BR-230, onde durante os anos de 1963 e 1970 ocorreram gradativas construções de instituições e conjuntos habitacionais, promovendo uma mudança no uso e ocupação do solo (Castro et al., 2015) e reduzindo a Mata Atlântica a um fragmento ocupado pela Unidade de Conservação (UC) denominada de Floresta
Nacional (FLONA) da Restinga de Cabedelo. É importante destacar que o crescimento da cidade limítrofe, João Pessoa, também contribuiu para a urbanização e a diminuição de áreas com vegetação do referido bioma (Stevens, 2014).

Portanto, estudos relacionados às variáveis microclimáticas entre o ambiente urbano e um dos últimos fragmentos de Mata Atlântica, a Floresta Nacional (FLONA) de Cabedelo, são escassos. Diante desse contexto, o estudo objetivou estudar o microclima urbano da cidade de Cabedelo-PB e sua relação com a FLONA de Cabedelo, partindo da hipótese da existência de um gradiente térmico entre a FLONA e a área urbanizada.

\section{Material e Métodos}

Caracterização da área de estudo

O município de Cabedelo localiza-se entre o Oceano Atlântico, à leste, com o Rio Paraíba, à oeste, e com João Pessoa-PB ao sul, entre as coordenadas geográficas $06^{\circ} 58^{\prime} 21^{\prime \prime}$ e $07^{\circ} 04^{\prime} 29^{\prime \prime} \mathrm{S}$ e 3450'18" e 34'51'20" W (Medeiros, 2012). Neste município está inserida a Floresta Nacional (FLONA) da Restinga de Cabedelo, uma Unidade de Conservação (UC) classificada, de acordo com a Lei $\mathrm{n}^{\circ} 9.985$ de 2000, como de uso sustentável, tendo como objetivos básicos os usos múltiplos dos recursos florestais e as pesquisas científicas, com ênfase em métodos para exploração sustentável de florestas nativas (Brasil, 2011). É conhecida como a Mata do Amém e possui uma área total de, aproximadamente, 115,82 ha, com altitude média de $10 \mathrm{~m}$ (Vasconcellos et al., 2005; Vicente et al., 2014).

O clima da região, segundo a classificação de Köppen (1918), é Am tropical chuvoso, com ocorrência de verão seco e quente, úmido durante todo o ano. Os dados climáticos da região, onde está inserida a área de estudo, apresentam temperaturas máximas de $31^{\circ} \mathrm{C}$ e mínimas de $21^{\circ} \mathrm{C}$, com média de $26^{\circ} \mathrm{C}$, para os anos de $1983 \mathrm{e}$ 2013 (Série Climatológica do INMET). Quanto à precipitação, a área de estudo apresenta ocorrência de duas estações definidas: uma seca e outra chuvosa, com seis meses de período chuvoso (março a agosto) e seis secos com estiagem (setembro a fevereiro) (INMET, 2016). 

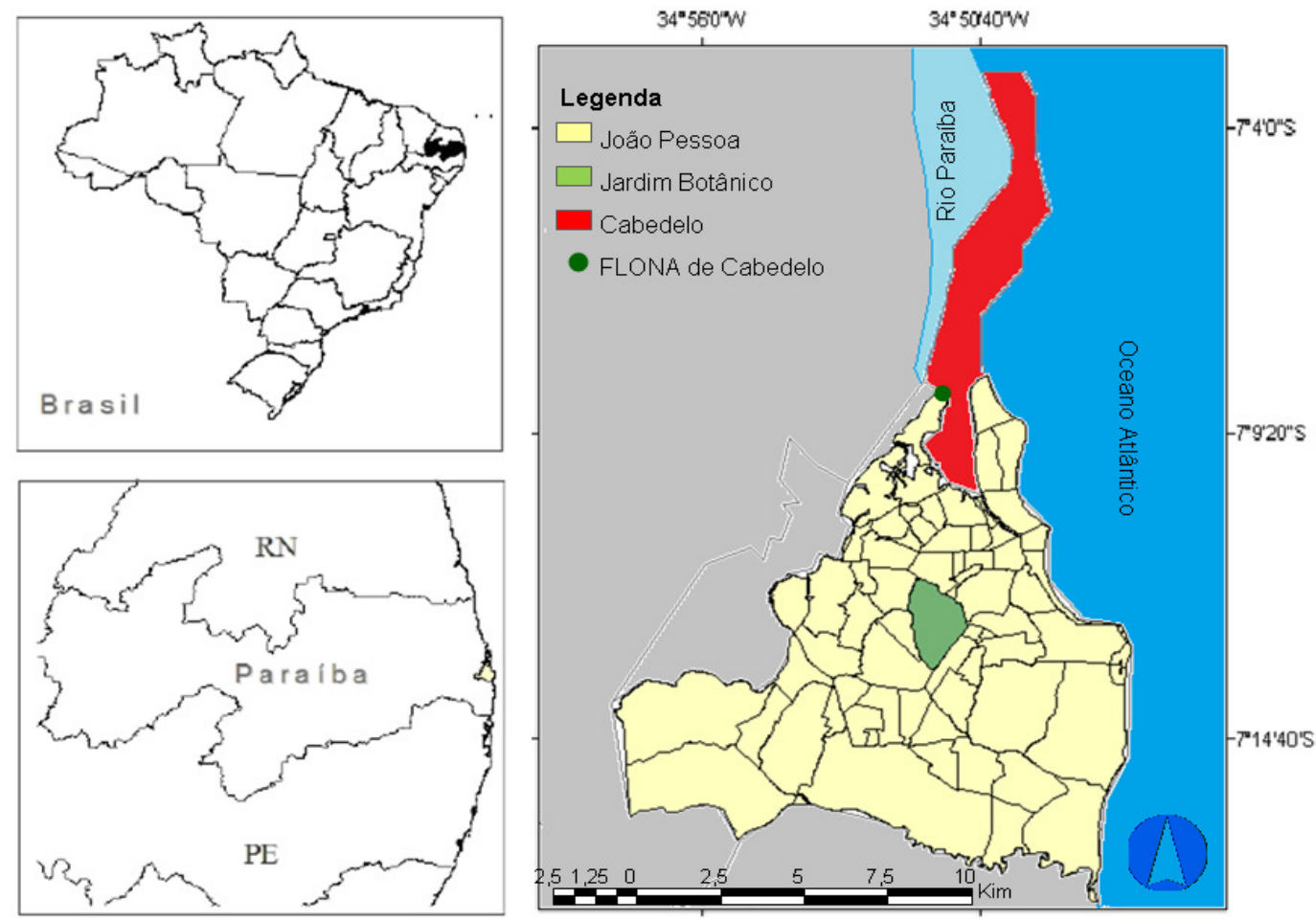

Figura 1. Localização geográfica da área de estudo (Floresta Nacional-FLONA), no município de CabedeloPB.

\section{Delimitação dos pontos experimentais}

Inicialmente foi realizado um levantamento bibliográfico, considerando a temática em questão, e documental, para a definição dos pontos a serem monitorados. Foram selecionados quatro pontos (Figura 2) para a instalação dos equipamentos para medição das variáveis microclimáticas (temperatura e umidade relativa do ar), sendo um na parte exterior e urbanizada da cidade de Cabedelo, localizado a $788 \mathrm{~m}$ da borda da FLONA, e outros três pontos situados na referida Unidade de Conservação, com um ponto na borda da mata, um no meio da mata (distante $50 \mathrm{~m}$ da borda) e outro no interior da mata (distante $150 \mathrm{~m}$ da borda), os quais compõem a FLONA de Cabedelo-PB.

Cada ponto de coleta de dados foi georreferenciado com GPS de navegação, utilizando-se o sistema de projeção UTM e Datum planimétrico SAD 69, para posterior espacialização no Sistema de Informação Geográfica. A Tabela 1 exibe os pontos experimentais, com as respectivas coordenadas geográficas.

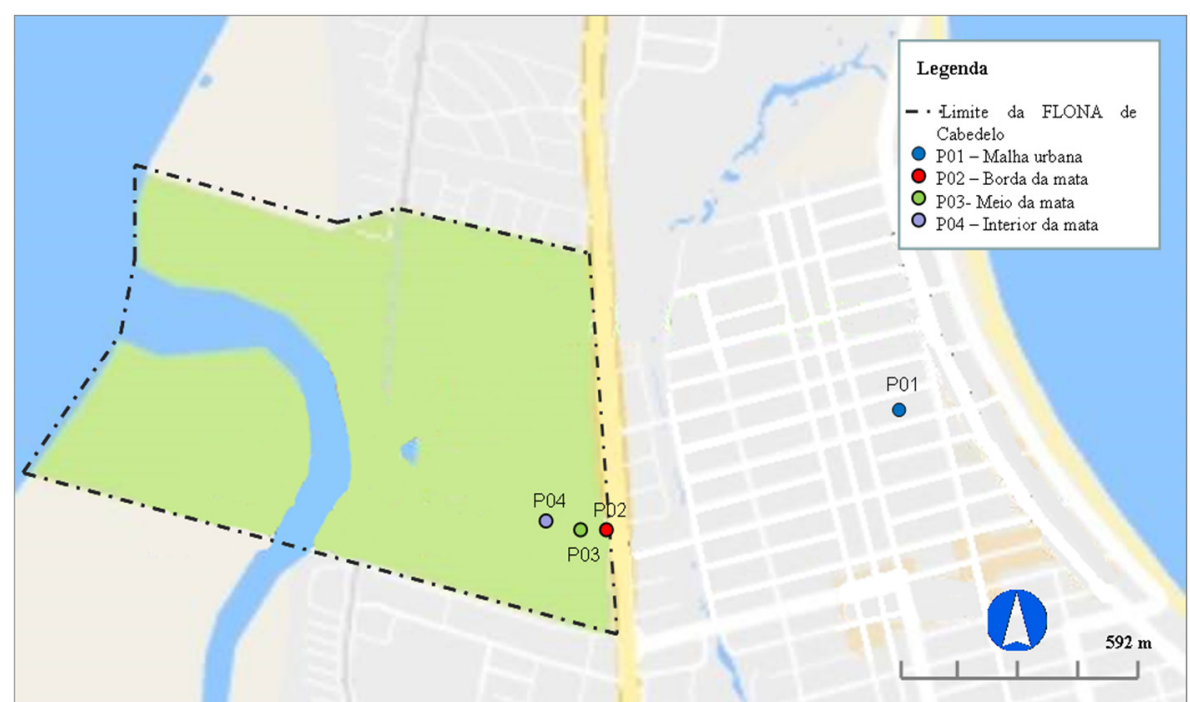

Figura 2. Localização dos pontos de monitoramento microclimático na Floresta Nacional (FLONA) de Cabedelo-PB. 
Tabela 1. Localização dos pontos experimentais, distribuídos na Floresta Nacional (FLONA) de Cabedelo, em coordenadas geográficas.

\begin{tabular}{|c|c|c|c|}
\hline Ponto & Localização & $\begin{array}{c}\text { Longitude } \\
\text { X }\end{array}$ & $\begin{array}{c}\text { Latitude } \\
\text { Y }\end{array}$ \\
\hline 1 & $\begin{array}{l}\text { Malha } \\
\text { urbana }\end{array}$ & $7^{\circ} 3$ '42”S & $34^{\circ} 50^{\prime} 37^{\prime \prime} \mathrm{O}$ \\
\hline 2 & $\begin{array}{c}\text { Borda da } \\
\text { mata }\end{array}$ & $7^{\circ} 4^{\prime} 1^{\prime \prime} \mathrm{S}$ & $34^{\circ} 51^{\prime} 1^{\prime \prime} \mathrm{O}$ \\
\hline \multirow[t]{2}{*}{3} & $\begin{array}{c}\text { Meio da } \\
\text { mata }\end{array}$ & $7^{\circ} 4^{\prime} 1^{\prime \prime} \mathrm{S}$ & $34^{\circ} 51^{\prime} 3^{\prime \prime} \mathrm{O}$ \\
\hline & $\begin{array}{l}\text { Interior da } \\
\text { mata }\end{array}$ & $7^{\circ} 3^{\prime} 60^{\prime \prime} \mathrm{S}$ & $34^{\circ} 51^{\prime} 6^{\prime \prime} \mathrm{O}$ \\
\hline
\end{tabular}

\section{Levantamento de dados}

A análise do clima urbano através de descrições físicas do espaço ocupado, neste estudo, foi fundamentada na metodologia apresentada por Monteiro (1976), Katzschner et al. (2002), Costa (2007) e Santos (2011). O método consiste em medições in loco e na análise das variáveis climáticas de temperatura e umidade relativa do ar.

Para a medição dessas variáveis microclimáticas, em cada ponto experimental foram instalados sensores Data Loggers $\mathrm{Hobo}^{\circledR}$ U10-003 (Resolução: Temperatura: $0.1^{\circ} \mathrm{C}$ em $25^{\circ} \mathrm{C}$ e $\mathrm{RH}: 0.07 \%$ em $25^{\circ} \mathrm{C}$ ), os quais foram programados para obter dados durante intervalos horários (Figura 3A). Os data logger foram protegidos em cabines meteorológicas plásticas de cor branca, as quais contem pequenas aberturas que permitem a livre circulação do ar, e evitam a incidência solar direta. Essas cabines foram apoiadas em tripés distando $1,5 \mathrm{~m}$ do solo (Figura 3B), constituindo-se em estações meteorológicas para medição de temperatura e da umidade relativa do ar.

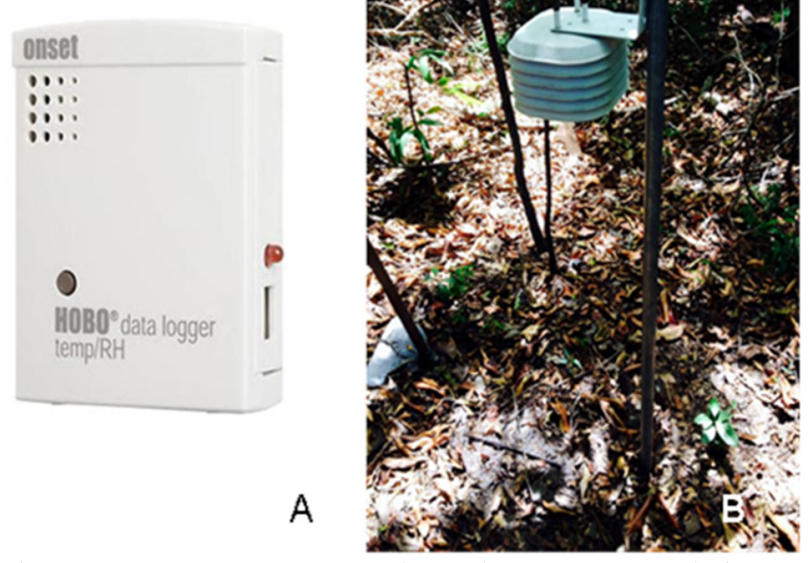

Figura 3. A. Sensores data logger; B. Abrigo plástico apoiado no tripé.

Tratamento dos dados
Os dados de temperatura e umidade relativa do ar, obtidos in loco, foram tratados através de estatística básica e descritiva e, posteriormente, tabulados através de uma planilha do programa Microsoft Excel ${ }^{\circledR}$.

As variáveis foram apresentadas através de tabelas e gráficos e avaliadas com relação às medidas descritivas, tais como valor máximo, valor mínimo, média e mediana. Posteriormente, realizou-se um comparativo entre as médias diárias e horárias dos pontos analisados e as duas áreas investigadas.

O teste de normalidade Shapiro-Wilk foi aplicado para avaliar se a distribuição era paramétrica ou não. Como resultou em dados nãoparamétricos, foi aplicado o teste de KruskalWallis $(\mathrm{p}<0,05)$, ambos executados no software BioStat ${ }^{\circledR}$ versão 7.0, para testar a hipótese.

\section{Resultados}

Descrição das amostras experimentais

As amostras experimentais foram denominadas da seguinte forma: P01 - Malha urbana, P02 - Borda da mata, P03 - Meio da mata e P04 - Interior da mata. Estas se caracterizaram da seguinte forma: P01 - Malha urbana: este ponto apresenta solo impermeabilizado com cerâmica, concreto, cobertura asfáltica, cobertura metálica e corpo hídrico - piscina e áreas permeáveis composto por vegetação arbórea, solo exposto e corpo hídrico - mar. P02 - Borda da mata: apresenta solo impermeabilizado com concreto, cerâmica, cobertura asfáltica e áreas permeáveis composto por vegetação arbórea e solo exposto. P03 - Meio da mata: possui solo impermeabilizado com concreto, cerâmica, cobertura asfáltica e áreas permeáveis composto por vegetação arbórea e solo exposto. P04 Interior da mata: este ponto é composto por solo impermeabilizado com concreto, cerâmica, cobertura asfáltica e áreas permeáveis composto por vegetação arbórea e solo exposto (Tabela 2). Os dados deletados estão apresentados na Tabela 2 , não devem estar no texto.

Tabela 2. Classes de cobertura do solo das amostras experimentais (\%), de acordo com o raio de $0,21 \mathrm{~km}^{2}$.

\begin{tabular}{lcccc}
\hline Cobertura & . P01 & P02 & P03 & P04 \\
\hline Cerâmica & 68,80 & 6,18 & 4,02 & 3,24 \\
$\begin{array}{l}\text { Concreto } \\
\text { Metálica }\end{array}$ & 19,10 & 20,54 & 10,68 & 9,97 \\
$\begin{array}{l}\text { Corpo hídrico } \\
\text { (mar) }\end{array}$ & 0,10 & 0 & 0 & 0 \\
$\begin{array}{l}\text { Corpo hídrico } \\
\text { (piscina) }\end{array}$ & 0,05 & 0 & 0 & 0 \\
Asfáltica & 5,87 & 3,63 & 2,80 & 2,70
\end{tabular}




\begin{tabular}{lcccc}
$\begin{array}{l}\text { Solo exposto } \\
\text { Vegetação }\end{array}$ & 4,78 & 10,95 & 8,10 & 7,51 \\
arbórea & 1,23 & 58,70 & 74,40 & 76,58 \\
\hline
\end{tabular}

Avaliação do gradiente de temperatura e umidade relativa do ar

De acordo com o tratamento dos dados no período avaliado, foi observado que o valor máximo de temperatura do ar ocorreu no P01, localizado na malha urbana do município de Cabedelo/PB. Esse ponto urbanizado apresentou uma média de $30,1^{\circ} \mathrm{C}$ para o período avaliado, seguido do P02 borda da mata (Tabela 3). Verifica-se que o valor mínimo ocorreu no P04 interior da mata $\left(22^{\circ} \mathrm{C}\right)$, tal ponto dista $150 \mathrm{~m}$ da borda (Tabela 3 ).

Deste modo, os dados revelam a existência de um gradiente térmico entre a FLONA e a malha urbana da cidade de Cabedelo/PB, que varia em $3,7^{\circ} \mathrm{C}$ entre as temperaturas mínimas das duas áreas. Esse dado é preocupante, pois a biodiversidade da FLONA pode ser afetada, tendo em vista que oscilações da temperatura mínimas podem afetar alguns microorganismos (de que modo?).

No que diz respeito às médias e medianas, também foram verificadas maiores temperaturas no P01 (malha urbana). Verificaram-se mudanças gradativas da borda em direção ao interior da mata, ou seja, os gradientes de temperatura podem ser observados entre $50 \mathrm{~m}$ e a $150 \mathrm{~m}$ de distância da borda em direção ao interior da mata (Tabela $3)$.

Tabela 3. Valores da temperatura relativa do ar durante o período de estiagem na Floresta Nacional (FLONA) de Cabedelo, PB. P01 = Malha urbana, $\mathrm{P} 02=$ Borda da mata, $\mathrm{P} 03=$ Meio da mata, P04 = Interior da mata.

\begin{tabular}{lcccc}
\hline Pontos & $\begin{array}{c}\text { Temperatura relativa do ar }\left({ }^{\mathbf{C}} \mathbf{C}\right) \\
\text { Valor } \\
\text { máximo }\end{array}$ & $\begin{array}{c}\text { Valor } \\
\text { mínimo }\end{array}$ & Média & Mediana \\
\hline P01 & 30,1 & 26,3 & 29,3 & 28,1 \\
P02 & 29,2 & 25,8 & 27,8 & 27,9 \\
P03 & 28,2 & 25,3 & 27,6 & 27,5 \\
P04 & 28,4 & 22,0 & 27,3 & 26,9 \\
\hline
\end{tabular}

$\mathrm{O}$ gradiente térmico, em relação á média da temperatura, variou em $1,5^{\circ} \mathrm{C}$, entre o $\mathrm{P} 01$ (malha urbana) e o P02 (borda da mata), em $0,2^{\circ} \mathrm{C}$ do P02 para o $\mathrm{P} 03$ (meio da mata) e em $0,3^{\circ} \mathrm{C}$ entre P03 e P04 (interior da mata). Entre o P04 (interior da mata) e o P01 (malha urbana), a diferença de temperatura foi de $2^{\circ} \mathrm{C}$ (Figura 4 ).

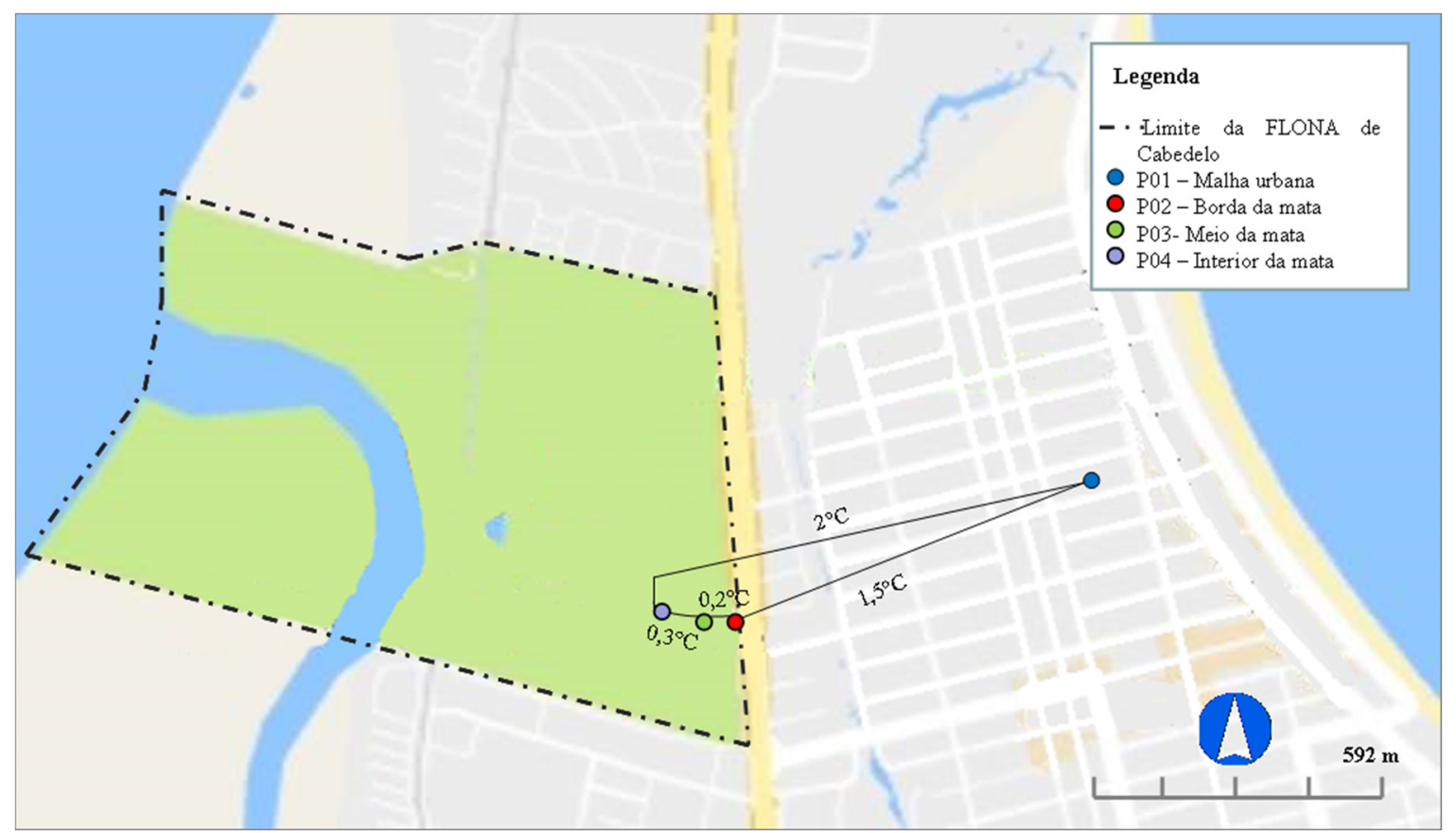

Figura 4. Esquema mostrando a variação de temperatura média entre os pontos experimentais na área de estudo (Floresta Nacional-FLONA de Cabedelo-PB).

No que se refere à umidade relativa do ar, verificou-se também um comportamento semelhante ao gradiente de temperatura. O valor máximo ocorreu no ponto P04 interior da mata, localizado na FLONA de Cabedelo, com 95,1\%, seguido do P03 meio da mata $(94,8 \%)$. Enquanto o valor mínimo ocorreu no P01 localizado na malha urbana de Cabedelo $(63,1 \%)$, seguido do ponto P02 localizado na borda da mata $(65,2 \%)$ (Figura 5).

As médias e as medianas demonstram maiores índices de umidade, nas áreas com maiores porcentagens de solo com vegetação arbórea (Tabela 2), ou seja, no ponto P03 (meio 
da mata) e P04 (interior da mata), havendo uma

(P01) e o meio da mata (P03) (Figura 5).

diferença média de 5,4\% entre a malha urbana
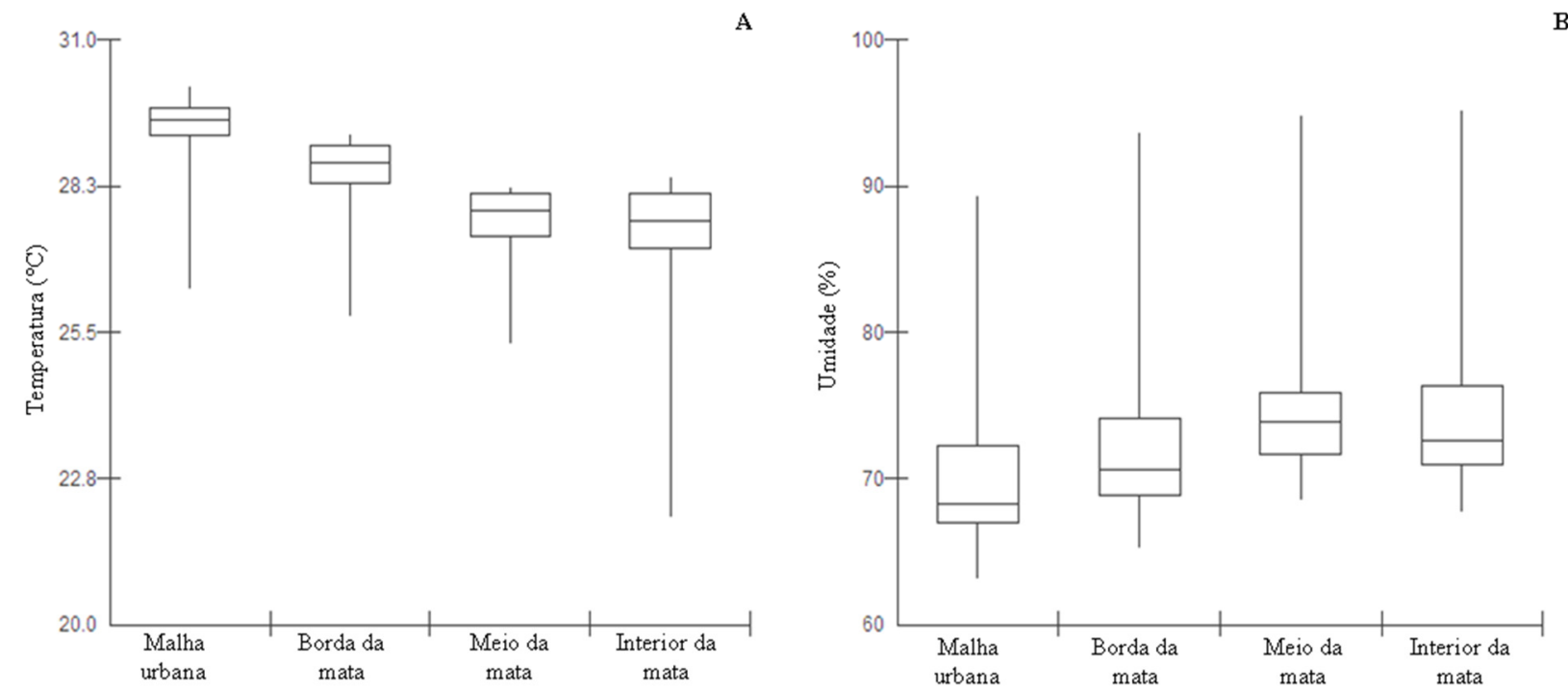

Figura 5. Box-plot das médias diárias da temperatura do ar (A) e umidade relativa do ar (B) dos pontos experimentais na área de estudo (Floresta Nacional-FLONA de Cabedelo-PB).

O box-plot também mostra mediana, a dispersão (primeiro e terceiros quartis), os valores máximos e mínimos de temperatura (Figura 5A) e a umidade (Figura 5B) de cada ponto monitorado.

A Figura 6 exibe o curso temporal das médias diárias da temperatura do ar e umidade relativa durante o período de estiagem, nos pontos monitorados. Evidenciando menores temperaturas e maiores umidades na medida em que segue sentido a malha urbana (P01) para o interior da mata (P04).
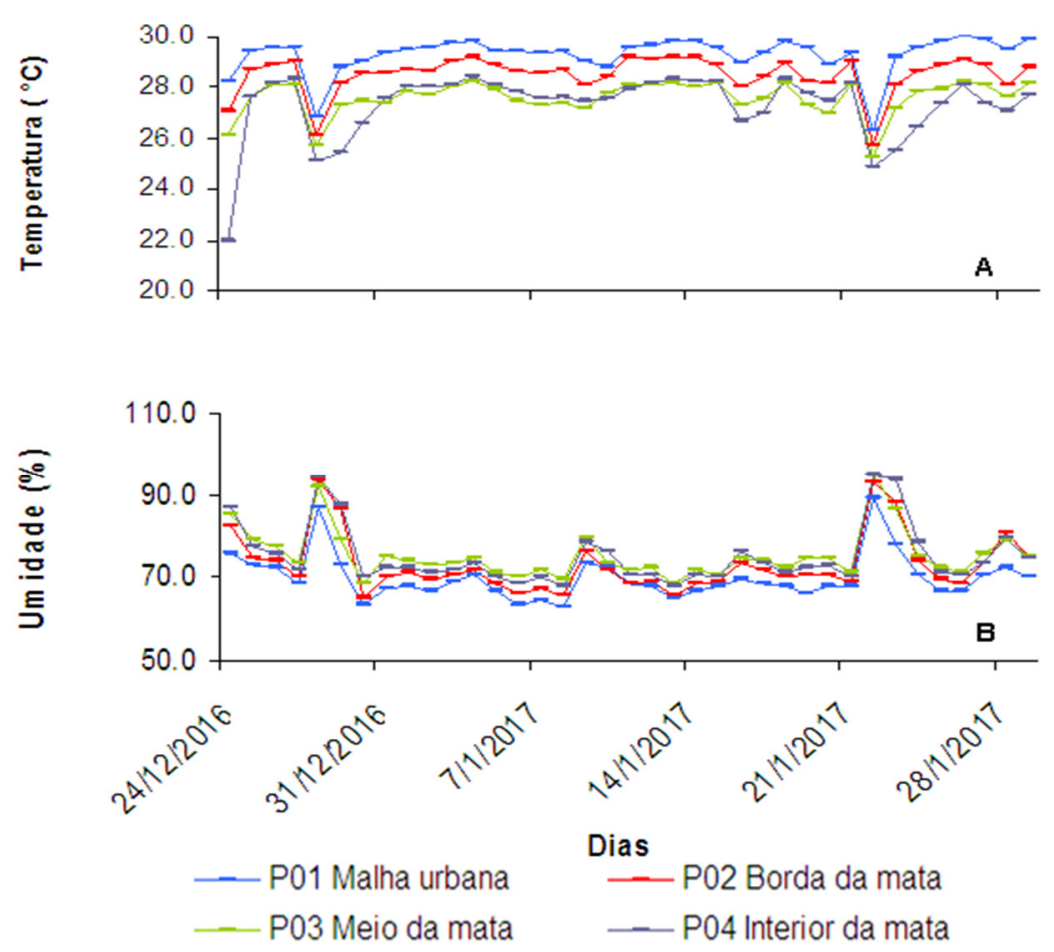

Figura 6. Curso temporal das médias diárias da temperatura do ar (A) e umidade relativa do ar (B) dos pontos experimentais na Floresta Nacional (FLONA) de Cabedelo-PB.

Avaliando o comportamento horário das variáveis microclimáticas dos pontos monitorados, durante o período de estiagem, verificou-se que as médias da temperatura do ar 
são mais baixas entre os horários que correspondem às $18 \mathrm{~h} 00 \mathrm{~min}$ e $06 \mathrm{~h} 00 \mathrm{~min}$, em face da ausência de incidência de radiação solar e redução da emissão de calor sensível emitido pela superfície. Em seguida, a temperatura do ar começa a aumentar até atingir o máximo em torno das $12 \mathrm{~h} 00 \mathrm{~min}$ e $14 \mathrm{~h} 00 \mathrm{~min}$, justamente quando ocorre a maior incidência de radiação solar e emissão de calor sensível. O aumento e a redução da temperatura do ar são acompanhados, respectivamente, pela redução/aumento da umidade relativa (Figura 7).
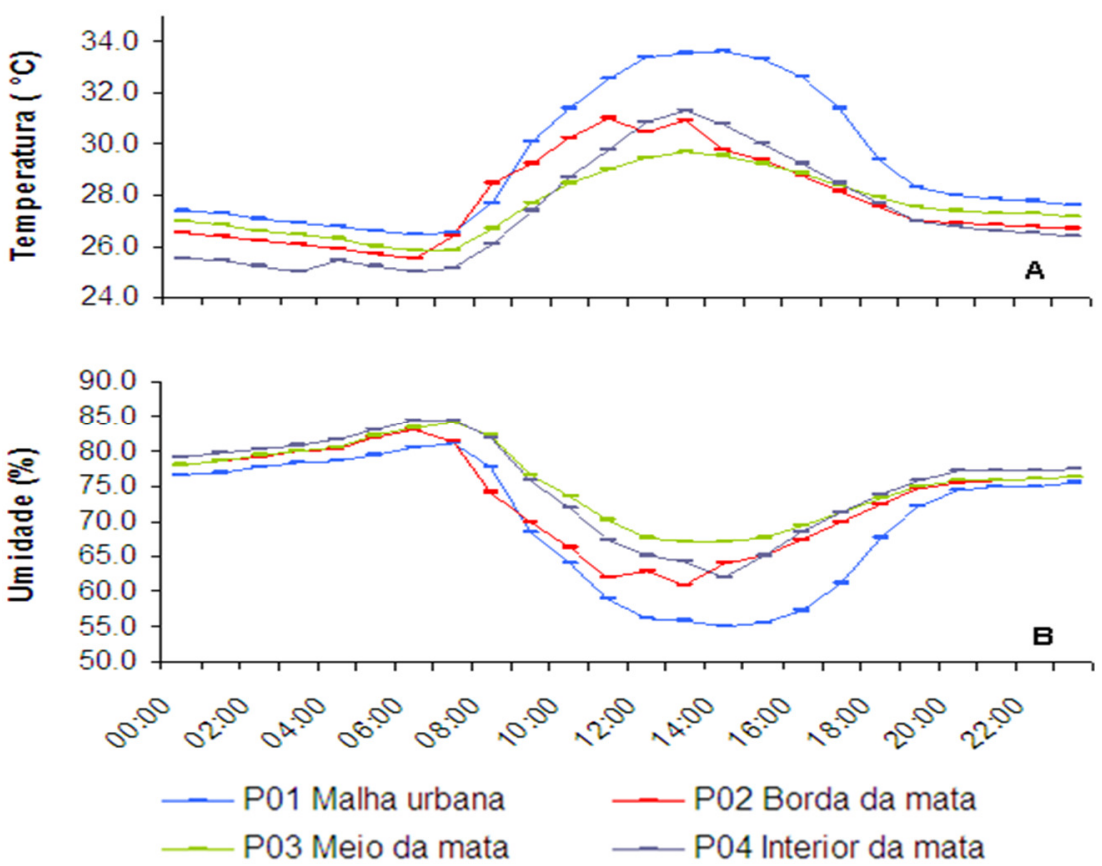

Figura 7. Curso temporal das médias horárias da temperatura do ar (A) e umidade relativa do ar (B) dos pontos experimentais na Floresta Nacional (FLONA) de Cabedelo-PB.

O teste de Kruskal-Wallis foi significativo, onde o valor do teste $\mathrm{H}$ (3 graus de liberdade $\mathrm{N}=148$ ), $\mathrm{o}$ valor de $\mathrm{p}<0,05$, mostrando que existe um gradiente de temperatura e umidade relativa do ar no sentido malha urbana da cidade de Cabedelo e interior da Unidade de Conservação Floresta Nacional da Restinga de Cabedelo.

Considerando esses resultados, é possível observar que a regulação do microclima é um dos mais importantes serviços ecossistêmicos proporcionados pela Flona de Cabedelo, sendo fundamental a manutenção de Unidades de Conservação em ambientes urbanos.

\section{Discussão}

Dacanal et al. (2010) encontraram resultados similares analisando fragmentos florestais urbanos inseridos em área de clima tropical. Os autores também verificaram que no interior desses fragmentos, a vegetação é densa e mais eficiente em atenuar a temperatura do ar gerando assim menores temperaturas.

Por outro lado, em ambientes urbanizados, os diversos parâmetros das construções civis, como orientação das edificações, densidade construída e albedo dos materiais, contribuem para o aumento das taxas de temperatura em regiões de climas quentes e úmidos (Abreu-Harbich et al., 2014).

Quanto às mudanças gradativas de temperatura da borda em direção ao interior da mata, Didham \& Lawton (1999) diagnosticaram a existência de um gradiente térmico entre $50 \mathrm{~m}$ e $100 \mathrm{~m}$, no sentido borda-interior de um fragmento florestal em região tropical. Esse gradiente ocorre devido aos diversos fatores abióticos e bióticos, deste último; um dos principais a influenciar é o grau da urbanização no entorno do fragmento (Sampaio, 2011; Monteiro et al., 2014; Freitas et al., 2016).

Buyadi et al. (2015) concluíram que as áreas urbanizadas podem influenciar na distribuição média da temperatura, tendo sido identificada uma diferença térmica de $1,3^{\circ} \mathrm{C}$ entre o fragmento vegetacional e a área construída. Tejas et al. (2011) encontraram uma diferença de $3 \%$ a $6 \%$ de umidade relativa do ar entre uma área urbanizada e uma arborizada. Shashua-Bar et al. (2011) e Zhang et al. (2016) afirmam que os 
resquícios de vegetação, com porte arbóreo, proporcionam melhores taxas de temperatura e umidade do ar, quando comparados com as áreas urbanizadas. Ecologicamente, a evapotranspiração vegetal influência na regulação dos parâmetros microclimáticos, favorecendo o aumento da umidade do ar e a diminuição da temperatura (Stan et al., 2016). Essa variação horária das variáveis microclimáticas foi observada por Xavier et al. (2009) em Cuiabá.

Segundo Takács et al. (2016), Ragheb et al. (2016) e Wang et al. (2017), a vegetação exerce papel imprescindível na redução do estresse térmico e, consequentemente, nas condições de conforto humano. Por outro lado, o processo de urbanização acelerado e, sem o devido planejamento ambiental, tem alterado as condições do clima urbano, influenciando diretamente no efeito de borda.

As menores temperaturas e maiores taxas de umidades relativas do ar foram registradas em pontos que estavam inseridos no fragmento de Mata Atlântica. Isso reforça a importância dessa vegetação, no que se refere aos serviços ecossistêmicos, especificamente, na amenização microclimática, em função da evapotranspiração e o sombreamento, que são as principais estratégias de controle da radiação solar pela interceptação dos raios solares e conversão da energia radiante em calor sensível, o que reduz as temperaturas de superfície dos objetos sombreados, aumentando a umidade relativa do ar e a ventilação.

$O$ ponto localizado na malha urbana apresentou maiores temperaturas e menores taxas de umidade relativa do ar. Isso ocorreu devido ao uso e ocupação do solo serem constituídos por materiais impermeáveis, o que contribui para o armazenamento de calor, reforçando a existência de um gradiente térmico da reserva em direção à malha urbana. Ocorreu também um gradiente térmico na interface interior da mata, em direção ao ambiente urbanizado, apresentando uma atenuação gradual da temperatura e um aumento da umidade relativa do ar à medida que se avança para o interior da FLONA de Cabedelo.

\section{Conclusão}

A hipótese desse estudo foi confirmada, corroborando estudos do campo termodinâmico realizados em regiões tropicais. As informações obtidas contribuirão para a implantação de ações de manejo e conservação da FLONA, tendo em vista que esta reserva florestal representa um serviço ambiental de amenização climática, imprescindível para a manutenção da qualidade de vida das populações que residem no seu entorno.

\section{Agradecimentos}

Os autores agradecem a Fabiano Gumier e todos que compõem a equipe gestora da Floresta Nacional (FLONA) de Cabedelo-PB, pela autorização e apoio técnico para a realização deste estudo.

\section{Referências}

ABREU-HARBICH, L. V.; LABAKI, L. C.; MATZARAKIS, A. 2014. Thermal bioclimatic in idealized urban street canyons in Campinas, Brazil. Theor. Appl. Climatol., v. 115, p. 333-340.

AMORIM, R. P. L. 2011. Análise da magnitude da influência climática de um remanescente de Mata Atlântica sobre o seu entorno urbanizado em clima quente-úmido. Dissertação de Mestrado, Universidade de Federal da Paraíba. João Pessoa, Paraíba, Brasil. 93p.

BRASIL. 2011. SNUC-Sistema Nacional de Unidades de Conservação da Natureza: Lei $\mathrm{n}^{\circ}$ 9.985, de 18 de julho de 2000. Brasília: MMA/SB.

BUYADI, S. N. A.; MOHD, W. M. N. W.; MISNI, A. 2015. Vegetation's role on modifying microclimate of urban resident. Procedia - Social and Behavioral Sciences, v. 202, p. 400-407.

CASTRO, A. A. B. C. et al. 2015. Interfaces rodoviário-urbanas no processo de produção das cidades: estudo de caso do contorno rodoviário de João Pessoa, PB, Brasil. Ambiente Construído. v. 15, n. 3, p. 175-199.

CHEN, J. et al. 1999. Microclimate in forest ecosystem and landscape ecology. BioScience., v. 49, n. 4, p. 288-297.

COSTA, A. D. L. 2007. O revestimento de superfícies horizontais e sua implicação microclimática em localidade de baixa latitude com clima quente e úmido. Tese de Doutorado, Universidade de Estadual de Campinas. Campinas, São Paulo, Brasil. 224p.

DACANAL, C.; LABAKI, L. C.; SILVA, T. M. L. 2010. Vamos passear na floresta! O conforto térmico em fragmentos florestais urbanos. Ambiente Construído, v. 10, n. 2, p. 115-132.

DIDHAM, R. K.; LAWTON, J. H. 1999. Edge structure determines the magnitude of changes in microclimate and vegetation structure in tropical forest fragments. Biotropica, v. 31, n. 1, p. 17-30. 
EWERS, R. M.; BANKS-LEITE, C. 2013. Fragmentation impairs the microclimate buffering effect of tropical forests. Plos One, v. 8, n. 3, p. 17.

FAHRING, L. 2003. Effects of habitat fragmentation on biodiversity. Annu. Rev. Ecol. Evol. Syst., v. 34, p. 487-515.

FREITAS, A. F.; SANTOS, J. S.; LIMA, R. B. 2016. Análise da variação microclimática em diferentes níveis de fragmentação. Revista Brasileira de Geografia Física, v. 9, n. 1, p. 226236.

$\begin{array}{lcc}\text { INSTITUTO } & \text { NACIONAL } & \text { DE } \\ \text { METEREOLOGIA. } & \text { Disponível } & \text { em: }\end{array}$
http://www.inmet.gov.br/portal/. Acesso em: 30 de out de 2016.

KATZSCHNER, L.; BOSCH, U.; ROTTEGEN, M. 2002. Behaviour of people in open spaces in dependency of thermal comfort conditions. In: International Conference on passive and low energy architecture. Anais France, James \& James Ltd., pp. 411-415.

MEDEIROS, M. C. 2012. A pesca artesanal na costa da Paraíba: um enfoque etnoictionológico. Dissertação de Mestrado, Universidade Federal de Campina Grande. Campina Grande, Paraíba, Brasil. 129p.

MCDONALD, R. I.; MARCOTULLIO, P. J.; GÜNERALP, B. 2013. Urbanization and global trends in biodiversity and ecosystem services. In: ELMQVIST, T. et al. (Ed.). Urbanization, biodiversity and ecosystem services: challenges and opportunities. Cap. 3, pp. 31-52.

MONTEIRO, C. A. F. Teoria e Clima Urbano. Tese de Doutorado, Universidade de São Paulo. USP. São Paulo, 1976. 236 p.

MONTEIRO, J. C. R. et al. 2014. Descrição da temperatura $\mathrm{e}$ umidade relativa do ar em diferentes localidades no bairro do Parque DezManaus/AM. Biota Amazônia, v. 4, n. 2, p. 20-27.

MYRES, N. et al. 2000. Biodiversity hotspots for conservation priorities. Nature, v. 403, n. 24, p. 853-858.

PETRISOR, A. I. et al. 2016. Assessing the fragmentation of the green infrastructure in Romanian cities using fractal models and numerical taxonomy. Procedia Environmental Science, v. 32, p. 110-123.

RAGHEB, A. A.; EL-DARWISH, I. I.; AHMED, S. 2016. Microclimate and human comfort considerations in planning a historic urban quarter. International Journal of Sustainable Built Environment, v. 5, p. 156-167.

RANTA, P. et al. 1998. The fragment Atlantic rain forest of Brazil: size, shape and distribution of forest fragments. Biodiversity and Conservation, v. 7, p. 385-403.

SAMPAIO, R. C. N. 2011. Efeito de borda em um fragmento de floresta estacional semidecidual no interior do estado de São Paulo. Dissertação de Mestrado, Universidade Estadual Paulista. Botucatu, São Paulo, Brasil. 83p.

SANTOS, J. S. 2011. Campo térmico urbano e a sua relação com uso e cobertura do solo em uma cidade tropical úmida. Tese de Doutorado, Universidade Federal de Campina Grande. Campina Grande, Paraíba, Brasil. 108p.

SAUNDERS, D. A.; HOBBS, R. J.; MARGULES, C. R. 1991. Biological consequences of ecosystem fragmentation: a review. Conservation Biology, v. 5, n. 10, p. 1832.

SHASHUA-BAR, L.; PEARLMUTTER, D.; ERELL, E. 2011. The influence of trees and grass on outdoor thermal comfort in a hot-arid environment. International Journal of Climatology, v. 31, p. 1498-1506.

STAN, F. I. et al. 2016. Study on the evaporation and evapotranspiration measured on the Căldăruşani Lake (Romania). Procedia Environmental Science, v. 32, p. 281-289.

STEVENS, P. O. 2014. Dinâmica da paisagem no geossistema do estuário do Rio Paraíba - extremo oriental das Américas: estimativas de perdas de habitat e cenários de recuperação da biodiversidade. Dissertação de Mestrado, Universidade Federal da Paraíba. João Pessoa, Brasil. 125p.

TAKÁCS, A. et al. 2016. Microclimate modification by urban shade trees - an integrated approach to aid ecosystem service based decisionmaking. Procedia Environmental Science, v. 32, p. 97-109. 
TEJAS, G. T.; AZEVEDO, M. G. F.; LOCATELLI, M. 2011. A influência de áreas verdes no comportamento higrotérmico e na percepção ambiental do citadino em duas unidades amostrais no município de Porto Velho, Rondônia, Brasil. REVSBAU, v. 6, n. 4, p. 15-34.

VASCONCELLOS, A. et al. 2005. Cupins de duas florestas de restinga do nordeste brasileiro. Iheringia, v. 95, n. 2, p. 127-131.

VICENTE, A. et al. 2014. Composição estrutural da vegetação lenhosa da restinga de Cabedelo, Paraíba. Revista de Geografia, v. 31, n. 1, p. 183196.

WANG, Y. et al. 2017. Thermal comfort in urban green spaces: a survey on a Dutch university campus. International Journal Biometeorological, v. 61, p. $87-101$.
XAVIER, A. L. et al. 2009. Variação da temperatura e umidade entre as áreas urbanas de Cuiabá. Engenharia Ambiental, v. 6, n. 1, p. 8293.

YI, Y. et al. 2016. Effects of urbanization on landscape patterns in a mountainous area: a case study in the Mentougou district, Beijing, China. Sustainability, v. 8, p. 1-14.

ZHANG, W.; ZHU, Y.; JIANG, J. 2016. Effect of the urbanization of wetlands on microclimate: a case study of xixi wetland, Hangzou, China. Sustainability, v. 8, n. 885, p. 1-13.

ZHOU, D. et al. 2014. Effects of urbanization expansion on landscape pattern and region ecological risk in Chinese coastal city: a case study of Yantai city. The Scientific World Journal, v. 2014, ID 821781, p. 1-9. 